\title{
GW23-e0181 ANTI-INFLAMMATORY EFFECT OF GINSENOSIDE RG1 ON CARDIOMYOCYTE INJURY INDUCED BY ADRIAMYCIN
}

doi:10.1136/heartjn-2012-302920a.130

Hongliang Kong, Hongliang Kong. The People's Hospital of Liaoning Province

Objectives Chronic inflammation have played an important role in heart failure (HF). Ginsenoside Rg1 (Gs-Rg1), stemming from Ginseng, is a kind of major pharmacologically active components. However, the effect of Gs-Rg1 in HF remains to being explored, let alone its mechanisms. Thus, the present study aimed at examining the effect of Gs-Rg1 in HF, and then further elucidates its effects on proinflammatory factor, such as tumour necrosis factor alpha $(\mathrm{TNF} \alpha)$, and nuclear transcription factor kappa B (NF-кB) basing on rat HF model induced by adriamycin, in vivo.

Methods Rat with adriamycin-induced HF were randomly divided into control group (age-matched health rat, $n=15$ ), HF group (ie, adriamycin group, $\mathrm{n}=15)$, Gs-Rg1 group (Gs-Rg1 intervention basing on HF, $\mathrm{n}=15)$, Gs-Rg1 was intraperitoneally administered according to body weight ( $4 \mathrm{mg} / 100 \mathrm{~g}$ ) once a day for 14 days. Left ventricular ejection fraction (LVEF) was estimated through echocardiographic examination, the above gene and the above protein was estimated through ELISA, real-time RT-PCR, western blot and Electrophoretic mobility shift assay.

\section{Results}

1. Gs-Rg1 significantly improved LVEF ( $p=0.005)$.

2. Both protein and mRNA of TNF $\alpha$ and TNFR-1 in the HF group were higher than that in the control group (all $\mathrm{p}<0.001$ ), which were markedly reduced by Gs-Rg1 (all $p<0.001$ ).

3. Gs-Rg1 augmented protein and mRNA of TNFR-2, which was lower in HF group than in control group.

4. Compared to HF group, Gs-Rg1 markedly inhibited the protein level of total-IKK $\alpha$, phospho-IKK $\alpha$, total-IKK $\beta$ and phosphoIKK $\beta$, including their IKK $\alpha$ phosporylation (ie, the ratio of phosphorylated to total protein) and IKK $\beta$ phosporylation (all $\mathrm{p}<0.001)$.

5. Treatment with Gs-Rb1 caused a significant increase in total$\mathrm{I} \kappa \mathrm{B}$ and $\mathrm{I} \kappa \mathrm{B}$, and a significant decrease in $\mathrm{I} \kappa \mathrm{B}$ phosporylation compared with HF group (all $\mathrm{p}<0.01$ ).

6. Gs-Rb1 markedly decreased total-NF-кB protein, phospho-NF$\kappa \mathrm{B}$ protein and NF-кB mRNA than that in HF group (all $\mathrm{p}<0.01)$. 


\section{ABSTRACTS}

Conclusions Gs-Rg1 may improve HF, which was mediated by proinflammatory factors, including a decrease in TNF $\alpha, N F-\kappa B$ and an increase in both TNFR-2 and IкB. 